\title{
Desarrollo de una Herramienta Software para la Vista de Información de la Arquitectura CIMOSA
}

\author{
Llanos Cuenca, Ángel Ortiz y Andrés Boza \\ Universidad Politécnica de Valencia, Centro de Investigación Gestión e Ingeniería de Producción, \\ Camino de Vera s/n, 46022 Valencia- España \\ (e-mail: Ilcuenca@cigip.upv.es, aortiz@cigip.upv.es, aboza@cigip.upv.es)
}

\begin{abstract}
Resumen
Este artículo presenta una herramienta de software para la vista de información de la arquitectura CIMOSA (Computer Integrated Manufacturing Open System Architecture). El objetivo es permitir a los usuarios del negocio modelar las necesidades de información con un conjunto finito de constructores de modelado, completando el ciclo de vida de modelos de empresa definido por CIMOSA. En el desarrollo se han seguido las fases de la metodología METRICA para el análisis, diseño, construcción e implantación de sistemas. Entre los resultados obtenidos destacan el modelado a nivel de propiedades o elementos de información y sus características asociadas. Se concluye que la herramienta favorece la alineación entre negocio y sistema de información, evita redundancia e inconsistencias y facilita la flexibilidad del sistema de información.
\end{abstract}

Palabras clave: integración empresarial, CIMOSA, vista de información, herramienta de software

\section{Development of a Software Tool for the Information View of the CIMOSA Architecture}

\begin{abstract}
This paper presents a software tool for the Information View of CIMOSA architecture (Computer Integrated Manufacturing Open System Architecture). The aim is to allow users to model the business information needs with a finite set of building blocks, completing the life cycle of models defined by CIMOSA. The METRIC methodology has been followed for the analysis, design, construction and implementation of the system. The results highlight the modeling of Information Elements and their associated characteristics. It is concluded that the tool promotes alignment between business and information system, avoiding redundancy and inconsistencies and provides flexibility for the information system.
\end{abstract}

Keywords: enterprise integration, CIMOSA, information view, software tool 


\section{INTRODUCCIÓN}

Las empresas necesitan modelar la funcionalidad y comportamiento en términos de procesos de negocio, actividades, eventos, la toma de decisiones, recursos, organización, etc. Estos modelos deben representar situaciones reales en la empresa, por lo que es necesario agilidad en el modelado para adaptarse a los constantes cambios. La necesidad de una infraestructura global de información de la empresa, que permita la cooperación intra e inter empresarial, se ha vuelto primordial para cualquier empresa que opere en un ambiente global (Interop, 2004).

La integración empresarial puede ser abordada desde distintas perspectivas. El comité europeo de estandarización (CEN) reconoce tres niveles de integración (Chen y Vernadat, 2004):

1) Integración entre dispositivos físicos, conexión de dispositivos, máquinas de control numérico, conexión de redes, etc.

2) Integración de aplicaciones, relacionado con la interoperabilidad del software y sistemas de bases de datos heterogéneos.

3) Integración de negocio, coordinación de las funciones que gestionan y controlan los procesos de negocio.

Los procesos de negocio son un factor clave para integrar una empresa (Aguilar-Savén, 2004). En este sentido se debe tener en cuenta que también es importante que la empresa pueda implementar y ejecutar la descripción de sus modelos de negocio. Diferentes técnicas han trabajado en esta línea como ARIS de SAP, DEM de Baan, CIMOSA, IEM, TOVE, IDEF, UML (Schekkerman, 2004; Cuenca et al., 2006). Sin embargo no todas abordan la empresa desde un punto integrado o no existe una herramienta completa para su implementación (Jonkers et al., 2004; Sheer, 1992). Por otra parte existe la dificultad de trasladar los modelos de negocio o modelos de empresa a modelos ejecutables y operables para la empresa (Johannesson, 2007). Según Vernadat (1996), el modelado de empresa se preocupa por la representación y la especificación de varios aspectos de las operaciones de la empresa, de los aspectos funcionales que describen qué cosas deberán ser hechas y en qué orden; de los aspectos de información que describen qué objetos son utilizados o procesados; de los recursos; y de los aspectos organizacionales. Berio y Vernadat (2001), lo definen como la representación de la estructura, organización y comportamiento de una entidad de negocio, para evaluar su ejecución y los flujos de control, información y material, con el propósito de aumentar la eficiencia.

Las arquitecturas de modelado de empresa proporcionan un conjunto de vistas de modelado. Cada arquitectura de modelado propone sus propias vistas de modelado, por ejemplo:

CIMOSA: Vistas de Organización, Recursos, Información y Función.

GRAI-GIM: Vistas del Sistema Físico, Decisional, de Información y Funcional.

PERA: Arquitectura de Organización y RR.HH., del Sistema de Información y del Equipo de Producción.

GERAM: Vistas de Organización, Recursos, Información y Función.

ARIS: Vistas de Función, Datos, Organización y Control.

Zachman: Datos, Función, Localización, Personas, Tiempo, Motivación.

TOGAF: Visión Arquitectónica, Arquitectura de Negocio, del Sistema de Información y Tecnológica.

Otras aproximaciones abordan el modelado para redes de empresa o redes colaborativas, como es el caso de los proyectos Virtual Enterprise/Virtual Organization ICT-based projects, el proyecto PRODNET o el modelo VITE (Camarinha-Matos y Hamideh, 2006), los cuales centran su estudio en las herramientas e infraestructuras como soporte a la colaboración.

Todas las arquitecturas analizadas incluyen en su marco de modelado la vista de información y sus relaciones con otras vistas (Tang et al., 2004; Cuenca et al., 2005; Boza et al, 2007). En algunas ocasiones se distingue entre el modelado de procesos de negocio y sus técnicas asociadas (Diagramas de flujo de proceso, IDEF0, Redes de Petri) y el modelado de sistemas de información (Diagramas de Flujo de datos, Diagramas Entidad Relación, UML), distinguiendo por tanto entre 
integración de la información e integración de procesos (Giaglis, 2001; Ni et al. 2007). Abordar estos conceptos de forma separada lleva a la no alineación entre negocio y sistema de información. Otro aspecto importante a tener en cuenta, es la comunicación entre modelos procedentes de diferentes herramientas y por tanto modelado con diferentes técnicas (Cuenca et al., 2006).

De entre las diferentes arquitecturas se ha seleccionado la arquitectura CIMOSA por ser ésta la más completa y formal en la definición de los elementos del marco de modelado.

\section{Arquitectura CIMOSA}

CIMOSA (Open System Architecture - Computer Integrated Manufacturing), es una Arquitectura de Sistemas Abiertos (AMICE, 1993) desarrollada dentro de una serie de proyectos ESPRIT (EP 688, 5288 y 7110) entre 1986 y 1994. El objetivo de este proyecto era elaborar una Arquitectura Abierta, definiendo un conjunto de conceptos y reglas para facilitar la construcción de futuros Sistemas CIM. Mediante un conjunto finito de conceptos de modelado, una empresa puede crearse su propio modelo de requerimientos $\mathrm{CIM}$, con la ventaja adicional de que ya existe un conjunto estandarizado de construcciones básicas y modelos parciales, que se pueden adaptar a las características de una empresa determinada mediante unos pasos perfectamente definidos, lo que permite un gran ahorro de tiempo y esfuerzo en la empresa. CIMOSA ha definido tres dimensiones para reflejar todos los conceptos requeridos para el modelado de una empresa (AMICE, 1993; Cuenca, 2006):

La dimensión de Generación o Bloques Constructivos que representa el grado de particularización que identifica el conjunto de modelos posibles. CIMOSA define los niveles Genérico, Parcial y Particular.

La dimensión de las Vistas o Panoramas que representa la estructura y comportamiento de un modelo considerando diversos aspectos de una empresa. CIMOSA define cuatro vistas, Función, Información, Recursos y Organización.

La dimensión de los Modelos de la Empresa que representa el ciclo de vida del modelo a partir de un punto de partida, que es la definición de los requerimientos del modelo. CIMOSA identifica el Nivel de Definición de Requerimientos (RDM), de Especificación de Diseño (DSM) y Descripción de la Implementación (IDM).

\section{Vista de Información de CIMOSA}

La vista de información permite la observación de la estructura de la información del negocio usada durante las operaciones de la empresa para los procesos de planificación, control y toma de decisiones. Las funciones de la empresa dependen de la en la información definida en las entradas y salidas. Para definir consistentemente, formalizar, estructurar y presentar esas entradas y salidas, CIMOSA define un conjunto de constructores que se reúnen en la vista de información.

ENTERPRISE OBJECT (EO): Describe generalidades, reales o abstractas, identificadas únicamente, que representan entidades del sistema de información, (es decir aquello que puede ser conceptualizado o pensado como parte de un todo). Puede contener otros Enterprise Objects (Sub-Objects) y/o Information Elements, descritos por mecanismos de abstracción.

OBJECT VIEW (OV): Describe uno o más aspectos de Enterprise Objects. Son un conjunto de propiedades de una o más Enterprise Objects desde un punto de vista dado. Está compuesto de Information Elements y/o Object Views.

INFORMATION ELEMENT (IE): Usados para representar elementos de información en una Enterprise Object u Object View. Cada elemento de información se define por un tipo de datos.

OBJECT RELATIONSHIP (OR): Describe las relaciones definidas entre dos Enterprise Objects del sistema de información. 
SPECIFIED OBJECT VIEW: Es un constructor usado para describir la especificación de la Object Views definida en el nivel de definición de requerimientos.

IMPLEMENTED OBJECT VIEW: Es un constructor usado para describir la implementación de las Object Views según su definición y especificación en los niveles anteriores.

La figura 1 representa gráficamente los bloques constructivos de la vista de información de CIMOSA y sus relaciones.

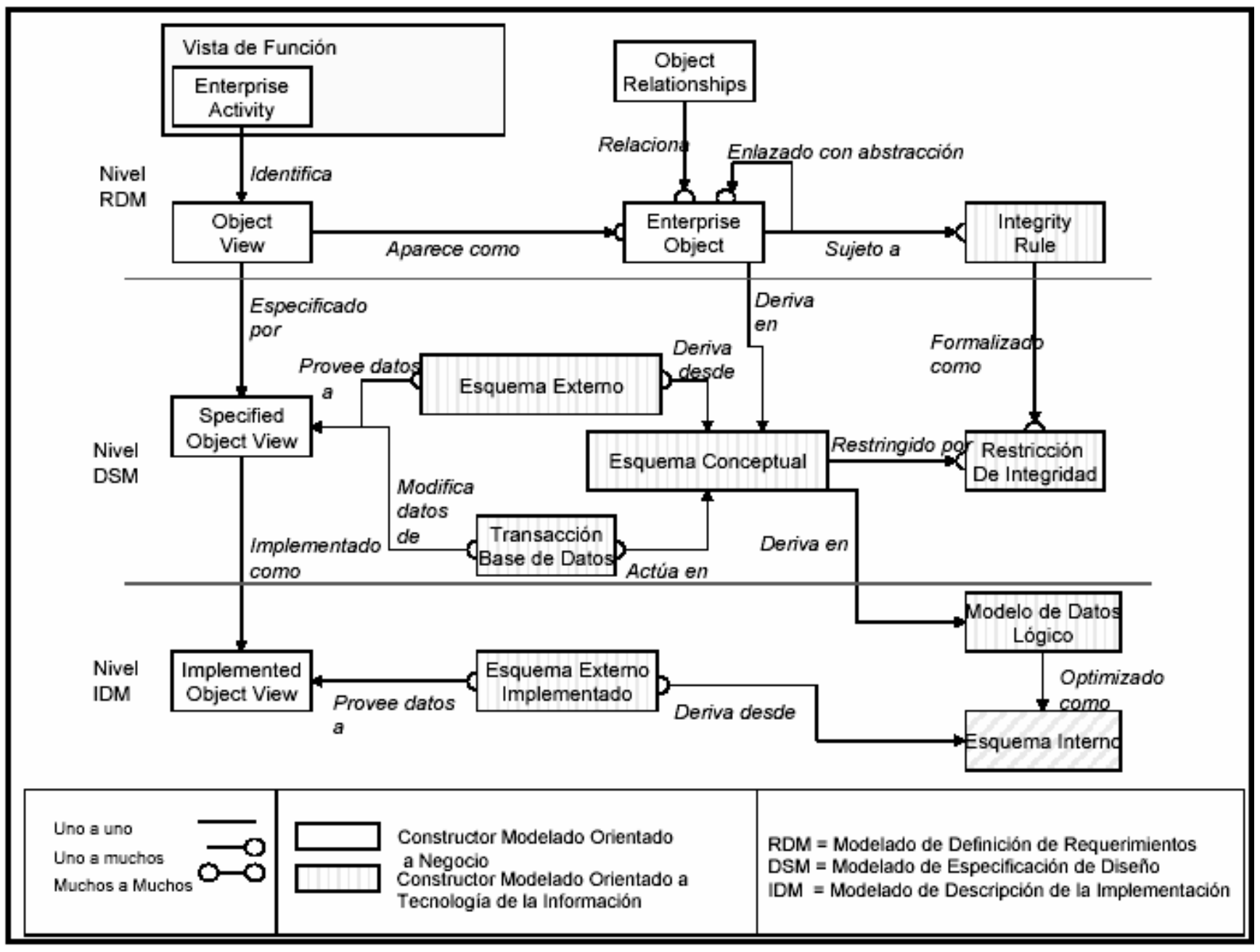

Fig. 1: Constructores para la Vista de Información en CIMOSA

CIMOSA, ha inspirado a desarrolladores de herramientas de modelado como, ARIS ToolSet, FirstSTEP, PrimeObject, o CimTool (Spadoni, 2004). Estas herramientas cubren aspectos de modelado de procesos de negocio pero están más centradas en la vista funcional, no completan el desarrollo de la vista de información desde el nivel de definición de requerimientos hasta la implementación. No soportan todos los elementos de modelado definidos por CIMOSA y tampoco generan los esquemas asociados a la implementación. También es difícil la comunicación entre ellas por la diferencias entre lenguajes de modelado utilizados (Spadoni, 2004; Mertins y Jockem, 2005).

El objetivo de este trabajo es presentar el desarrollo de una herramienta de modelado para la Vista de Información, dentro del marco de la arquitectura CIMOSA. El objetivo principal de la herramienta, es permitir a los usuarios del negocio modelar las necesidades de información con un conjunto finito de constructores de modelado, completando el ciclo de vida de Modelos de Empresa definido por CIMOSA, para los niveles de Definición de Requerimientos, Especificación de Diseño y Descripción de la Implementación, a partir de los requerimientos de información introducidos en forma de IE, OV 
y EO. De esta forma se consigue mejorar la alineación entre el modelo de negocio y el sistema de información. En los siguientes apartados se expone la Metodología seguida para su implementación y aplicación al desarrollo de la herramienta desarrollada, la implantación en una empresa del sector cerámico y por último los resultados obtenidos y conclusiones del trabajo.

\section{METODOLOGÍA DE DESARROLLO}

La Metodología seguida en el proceso de desarrollo de la herramienta ha sido METRICA, esta metodología es una guía formal, aunque flexible para el análisis, diseño, construcción e implantación de sistemas de información, empleando conceptos y técnicas de ingeniería de sistemas de información y tecnologías de la información. METRICA contempla el desarrollo de sistemas de información para distintas tecnologías cubriendo aspectos de gestión que aseguran el cumplimiento de sus objetivos en términos de calidad, presenta un enfoque orientado al proceso, incluyendo las siguientes fases (METRICA, 2007; Ochoa et al., 2006):

Fase1: Análisis de Sistemas, donde se define el ámbito y alcance del proyecto junto con el análisis de requerimientos.

Fase 2: Diseño de Sistemas, donde se establece el diseño técnico del sistema

Fase 3: Construcción de Sistemas, donde se desarrollan los componentes del sistema

Fase 4: Implantación de Sistemas, donde se realizan pruebas, implantación y aceptación del sistema

Se describe a continuación la aplicación de METRICA en el contexto de la herramienta para la Vista de Información de CIMOSA.

\section{FASE 1: Análisis de Sistemas}

El ámbito y alcance del proyecto se centra en el desarrollo de una herramienta software para la vista de información de CIMOSA, su funcionalidad permite modelar desde la definición de requerimientos por parte del usuario, hasta la descripción de la implementación. El análisis de requerimientos tiene como punto de partida el estudio de la arquitectura CIMOSA y en concreto de los constructores definidos en la Vista de Información. A partir de las plantillas o templates que CIMOSA define para cada constructor de la vista de información y las relaciones entre ellos, se han extraído los requerimientos de información necesarios para la herramienta. Como requerimiento funcional, la herramienta generará de manera semi-automática los diferentes esquemas para el Nivel de Especificación de Diseño y de Descripción de la implementación de CIMOSA.

\section{Fase 2: Diseño de Sistemas}

Para el Diseño Técnico del sistema, se toma como punto de partida el Paradigma de Modelado de CIMOSA. Este Paradigma se basa en tres enfoques de modelado, uno para cada Nivel de Modelado de CIMOSA.

Para el Nivel de Modelado de Definición de Requerimientos, CIMOSA propone hacer uso de un lenguaje que pueda ser fácilmente entendido por los usuarios, no familiarizados con la informática. El lenguaje elegido se basa en los constructores Enterprise Object, Object View, Object Relationship, Information Element y Reglas de Integridad. Para CIMOSA la definición de Enterprise Object y Object Relationship no es obligatoria, sino que se deja libertad al usuario. Sin embargo los autores consideran necesaria su modelización para facilitar la derivación del esquema conceptual del subyacente sistema de información, tal y como se define en el Nivel de Modelado de Especificación de Diseño. Para garantizar la consistencia entre modelos se ha optado por hacer obligatoria su definición, garantizando así la completa definición por parte del usuario.

Para el Nivel de Modelado de Especificación de Diseño, CIMOSA sigue una aproximación a un modelo de datos semántico, la metodología Entidad-Relación-Atributo (ERA). A partir de ahí las Restricciones en información y datos se han descrito por el significado de las Restricciones de Integridad derivadas de las Reglas de Integridad, por otra parte, las Transacciones describen las operaciones ejecutadas en el Esquema Conceptual para la manipulación de datos (es decir, los datos dinámicos). Las transacciones 
son de hecho, especificaciones de la Vista de Información de las operaciones funcionales de almacenamiento de datos definidas en la Vista de Función.

Para el Nivel de Modelado de Descripción de la Implementación se hace uso de un modelo de datos clásico (el modelo relacional) para describir el Esquema Interno que da soporte a la descripción de la implementación de las Object Views. Este esquema se obtiene usando dos procesos sucesivos de derivación del esquema, uno da como resultado el Modelo de Datos Lógico y el segundo para producir una versión implementable (es decir, ejecutable) de la estructura de los datos del Esquema Interno. Para ello se utiliza un lenguaje de datos tipo SQL.

\section{Fase 3: Construcción de Sistemas}

La implementación de la herramienta se ha llevado a cabo siguiendo las restricciones dadas por CIMOSA respecto a los elementos de la vista de información, definidas en la fase de diseño. Se ha desarrollado una aplicación Cliente/Servidor construida con la herramienta de desarrollo Delphi 5. Trabaja sobre una base de datos INTERBASE pero todos los accesos son de tipo SQL estándar, por lo que permite puede atacar cualquier servidor de datos SQL. Además de poder trabajar sobre diferentes bases de datos, la aplicación soporta múltiples proyectos y múltiples usuarios (identificados con Login y Pasword). Cada proyecto es independiente de los otros y puede estar completo en cualquier estadio de su desarrollo. La generación de los diferentes modelos es automática, la herramienta soporta las técnicas de transformación necesarias. No es solo una herramienta de modelado sino una herramienta CASE (Computer Aided Software Engineering) basada en CIMOSA, cumpliendo así con los requerimientos funcionales.

\section{Fase 4: Implantación de Sistemas}

Una vez desarrollada la herramienta siguiendo las fases de la metodología anteriormente descrita se procedió a su implantación en una empresa del sector cerámico, lo que permitió su validación. Se muestra a continuación parte del proyecto introducido por la empresa.

Desde la pantalla principal de la aplicación se controla el uso, creación y modificación de los proyectos. Para cada proyecto la aplicación permite acceder a cada uno de los niveles de modelado soportados por CIMOSA (Requerimientos, Diseño e Implementación).

En el nivel de definición de requerimientos, el usuario de la empresa dará de alta los siguientes elementos:

Propiedades. Estas propiedades se corresponden con los Elementos de Información definidos en CIMOSA, a partir de ellas se construirán las Objects Views (OV) y los Enterprise Objects (EV). Una propiedad está identificada unívocamente por su nombre, siendo sus características principales el tipo de datos, tamaño de los datos, cardinalidad y restricciones de identificación (clave y único) y de valor (requerido, operador y valor). Desde el mantenimiento de propiedades se puede consultar a qué OV y/o EO está asignada.

Object Views: Desde la opción Mantenimiento de OV y a partir de las propiedades introducidas anteriormente, se construyen las OV. Las características de una OV vendrán marcadas por las restricciones definidas sobre sus propiedades. A una OV se pueden asignar propiedades u otras OV, creando una estructura Padre-Hijo entre OV. La aplicación comprueba que no se formen ciclos en estas estructuras.

La figura 2 muestra el listado de propiedades previamente introducidas y la pantalla de introducción de OV. El usuario seleccionará las propiedades que formarán parte de la OV. Para cada OV se incluye también información de otras vistas con las que tiene relación, en este caso la Vista de Organización, donde se indica las celdas de organización y unidades de organización que tiene responsabilidad sobre esa OV. También se indica el tipo de OV, física o de información. En este caso se ha creado la Object View "Datos_Cliente". 


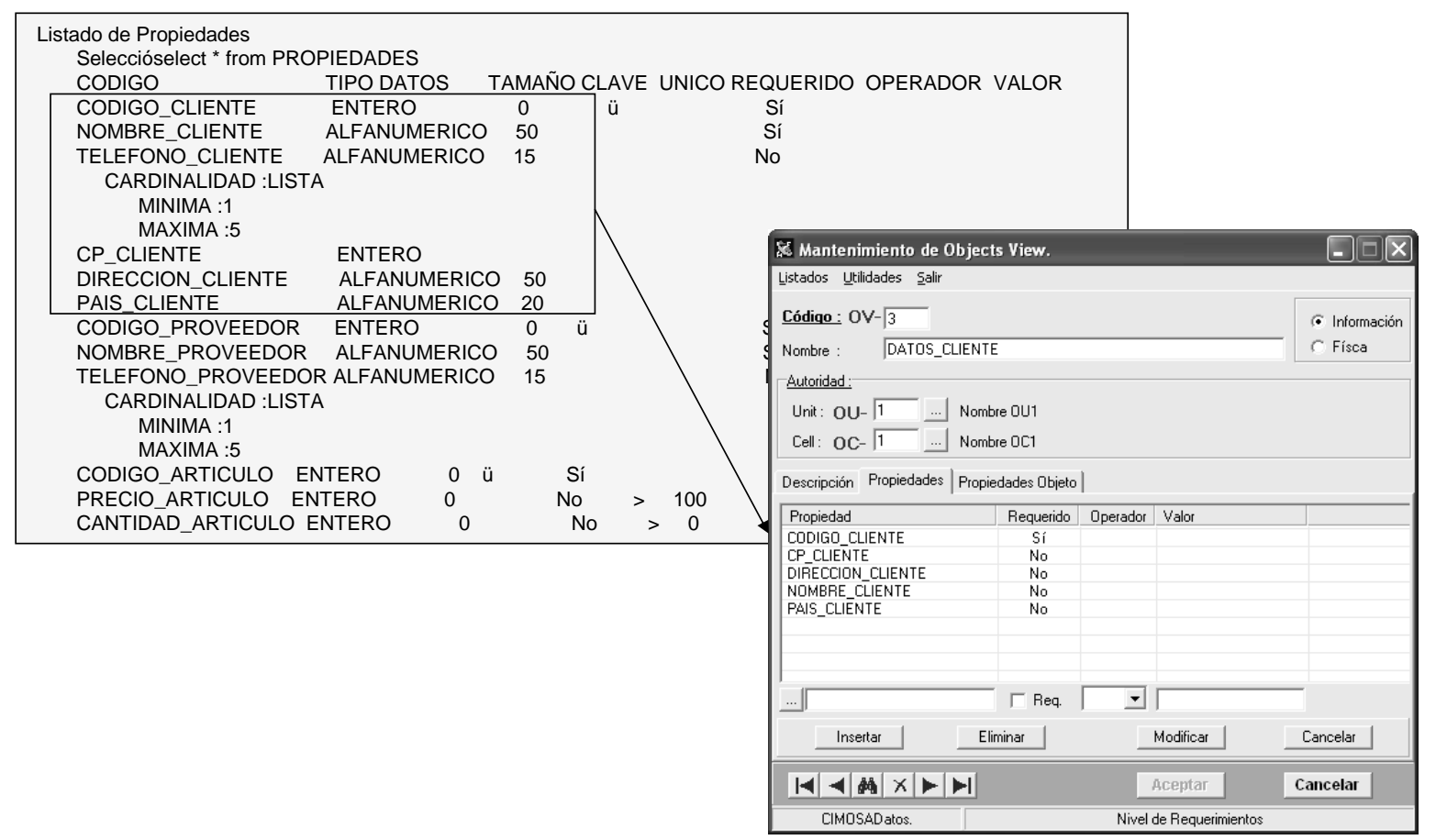

Fig. 2: Asignación de Propiedades a la Object View

Enterprise Objects (EO) y Relaciones entre EO se definirán también en el nivel de definición de requerimientos. Una vez completada toda la información la aplicación realiza un chequeo de la consistencia y se estará en disposición de pasar a la siguiente fase. En esta fase se creó la EO "Clientes"

En la fase de Especificación de Diseño a partir de la información introducida en la Definición de Requerimientos y de las reglas de transformación introducidas en la herramienta, se genera automáticamente con la opción "Obtener Modelo" el Esquema Entidad-Relación (EER). El EER consta de cuatro gráficos que muestran las Entidades, Relaciones, Generalidades y Vistas asociadas a la información introducida. La figura 3 muestra un ejemplo de las Entidades generadas. Para la Enterprise Object "Cliente" se ha creado una entidad y las propiedades introducidas han pasado a ser atributos de esa entidad.

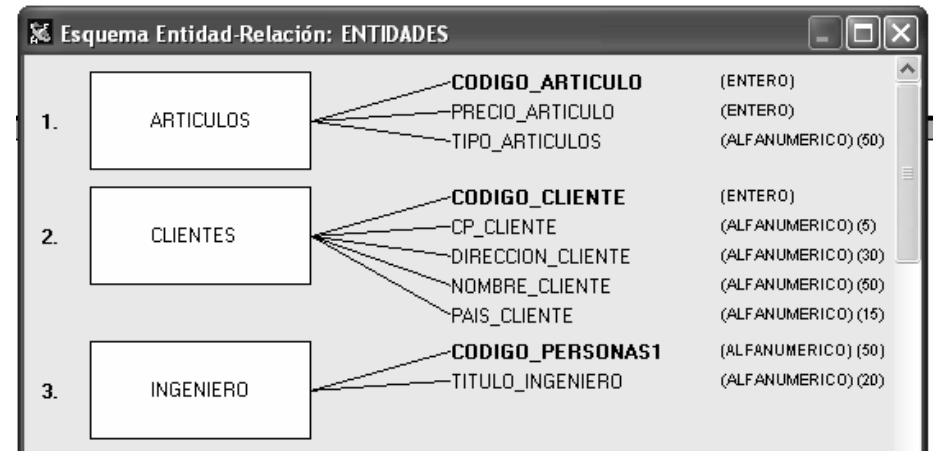

Fig. 3: Esquema Entidad Relación

Para terminar, en la fase de Descripción de la Implementación, se analizan todas las entidades, las generalidades y las relaciones del EER para transformarlas en Relaciones del Esquema Relacional. Estas Relaciones son la antesala de lo que serán las tablas de una base de datos SQL. El sistema genera un listado de excepciones con aquellos problemas que haya podido detectar durante el proceso de transformación. La última opción del nivel de descripción de la implementación es la obtención de un scrip para la creación de nuestra base de datos SQL. Este scrip será el resultado de toda la información y la semántica introducida por la empresa y modelada a través de los diferentes niveles y esquemas de representación. La figura 4 muestra como, con la información del esquema relacional se ha generado el scrip, a partir del cual se crearán las tablas en la base de datos. 


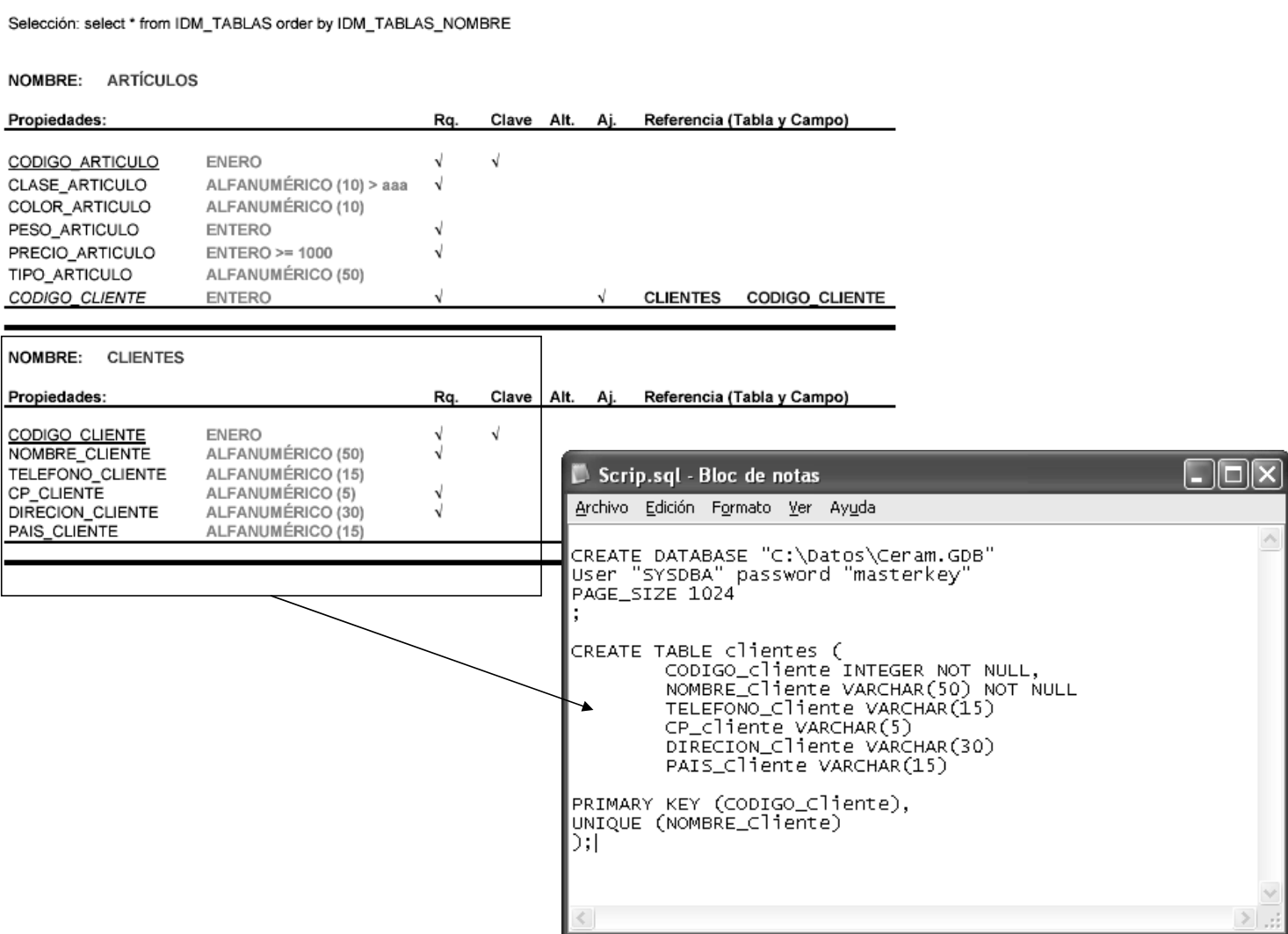

Fig.4: Listado de relaciones y scrip generado para implementación

Por otra parte ya que no todos los gestores de bases de datos tienen idéntica sintaxis para la definición de tablas, el scrip puede ser editado y modificado por el usuario.

\section{RESULTADOS Y DISCUSIÓN}

Los resultados obtenidos con la aplicación desarrollada son:

Listado de propiedades como elementos de información de CIMOSA. Al igual que ocurre con la herramienta CIMTool, se pueden asociar propiedades a las Object Views sin embargo la información asociada a cada propiedad en esta herramienta está limitado sólo al tipo de datos. En la herramienta presentada en este artículo se ve como además del tipo de datos se introduce también el tamaño de los datos, cardinalidad y restricciones de identificación y de valor. El resto de herramientas basadas en CIMOSA, FirstStep y PrimeObject no soportan este nivel de detalle.

Asociación de Propiedades a Object Views. A diferencia del resto de herramientas de modelado, las propiedades se introducen de forma independiente a la lista de Object Views y posteriormente se asocian, esto permite eliminar inconsistencias y redundancias en la información. Se evita que diferentes usuarios puedan denominar de forma distinta a una misma propiedad aunque se refieran al mismo concepto.

Generación del Esquema Entidad-Relación, Modelo Relacional y Scrip SQL. A partir de las reglas de transformación introducidas en la herramienta los datos introducidos no quedan como mera información almacenada difícil de manejar y con poca utilidad, sino que al soportar la herramienta todos los niveles de modelado de un sistema CIM, como propone CIMOSA, se genera el fichero resultado que permitirá la construcción de la base de datos que soporte el dicho sistema CIM. Este aspecto no quedaba resuelto en las otras herramientas de modelado anteriormente mencionadas.

Generación de listados. La información introducida puede ser listada y exportada a los formatos rtf y HTML. 


\section{CONCLUSIONES}

En este trabajo se ha presentado una herramienta software para la vista de información de CIMOSA. La herramienta, permite a los usuarios del negocio, modelar mediante un conjunto finito de constructores de modelado, el ciclo de vida de Modelos de Empresa definido por CIMOSA, para los niveles de Definición de Requerimientos, Especificación de Diseño y Descripción de la Implementación.

Las aportaciones que ofrece la herramienta son:

Soporte a la vista de información y por tanto el sistema de información asociado bajo el prisma integrador de la arquitectura CIMOSA. Se establecen relaciones con la Vista de Función y la Vista de Organización.

Permite a los usuarios del negocio introducir ellos mismos los requerimientos de información bajo su visión del problema pero sin perder la visión global del problema. De esta forma se consigue mejorar la alineación entre el modelo de negocio y el sistema de información.

La información se introduce manteniendo una independencia física y lógica de los datos. De esta forma se consigue organizar la información independientemente de las aplicaciones que los vayan a usar y de los ficheros en los que vayan a almacenarse.

La herramienta soporta los niveles de especificación del diseño y descripción de la implementación, así como las reglas de transformación necesarias de manera automática. De esta forma se mantiene la integridad semántica de los datos. Por otra parte cualquier cambio en la definición de requerimientos se traduce rápidamente a nivel de diseño e implementación, lo que favorece la flexibilidad del sistema de información.

Las restricciones sobre los Elementos de Información, Object Views y Enterprise Object evita redundancia y por tanto inconsistencias en la información. De esta forma se garantiza una base datos única y consistente.

Soporta el ciclo de vida completo desde la definición de requerimientos hasta la implementación, lo que hace que esta herramienta no sea sólo una herramienta de modelado de la información sino que sea también una herramienta de soporte a la implementación del sistema de información.

\section{AGRADECIMIENTOS}

Este trabajo ha sido realizado en el marco del proyecto CICYT - DPI2004-02594. Interoperabilidad de Procesos Extendidos.

\section{REFERENCIAS}

Aguilar-Saven, R.S.; Business process modelling: Review and framework. International Journal of Production Economics: 90 (2), 129-149. (2004)

AMICE. CIMOSA: Open System Arquitecture for CIM, 2nd. Revised and extended version. SpringerVerlag, Berlin. (1993)

Berio G. y F.B. Vernadat; Enterprise modelling with CIMOSA: functional and organizational aspects. Production Planning and Control: 12(2), 128-136 (2001)

Boza A., F. Alarcón, E. Vicens y M.M. Alemany; Propuesta de Marco Conceptual para el Modelado del Proceso de Planificación Colaborativa de una Red de Suministro/Distribución (RdS/D). Visión Informacional. International Conference on Industrial Engineering and Industrial Management, 1, 110, September 5-7, (2007) Madrid (Spain) 
Camarinha-Matos L. y A. Hamideh; Towards a Reference Model for Collaborative Networked Organizations. Information Technology For Balanced Manufacturing Systems. 193-202 Springer (2006)

Cuenca LI., A. Ortiz y A. Boza; Arquitectura de Empresa. Visión General, IX Congreso de Ingeniería de Organización. 1-8 (2005) Gijón (España) (fecha de consulta Junio 6, 2006; http://cio2005. uniovi.es/ cio2005/VerDocumento.do?id=96\&tipo=final)

Cuenca LI., A. Ortiz y F.B. Vernadat; From UML of DFD models to CIMOSA partial models and enterprise components. International Journal of Computer Integrated Manufacturing: 19(3), 248-263 (2006)

Chen D. y F.B. Vernadat; Standard on enterprise integration and engineering-state of the art. Int.J. Computer Integrated Manufacturing: 17(3), 235-253 (2004)

Giaglis G.; A Taxonomy of Business Process Modeling and Information Systems Modeling Techniques. The International Journal of Flexible Manufacturing Systems: 13 (2) 209-228 (2001)

Interop. Interoperability Research for Networked Enterprises Applications and Software NE INTEROP - IST Network of Excellence, www.interop-noe.org (2004)

Jonkers H. y otros cinco autores; Concepts for Modeling Enterprise Architectures. International Journal of Cooperative Information Systems: 13(3), 257-287 (2004)

Johannesson P.; The Role of Business Models in Enterprise Modelling- Conceptual Modelling in Information Systems Engineering. Springer Berlin Heidelberg, 123-140 (2007)

Mertins K. y R.Jockem; Architectures, methods and tools for enterprise engineering. Int. J. Production Economics: 98(2), 179-188 (2005)

METRICA. Ministerio de Administraciones Públicas de España - Consejo Superior de Informática. (2007). (fecha de consulta Abril 10, 2007; http://www.csi.map.es/csi/metrica3/index.html)

Ni Q., Lu W., Yarlagadda P. y Ming X.; Business information modeling for process integration in the mold making industry. Robotics and Computer-Integrated Manufacturing: 23(2), 195-207 (2007)

Ochoa, M.; P. Britos; y R. García-Martínez. Una Protofase de Entendimiento del Negocio para Metodologías de Desarrollo de Sistemas. XII Congreso Argentino de Ciencias de la Computación. San Luis. Argentina. (2006) (fecha de consulta Abril 20, 2007; http://www.itba.edu.ar/capis/webcapis/RGMITBA/comunicacionesrgm/CACIC-2006-Articulo-695.pdf)

Scheer, A.-W.; Architecture of Integrated Information Systems: Foundations of Enterprise Modeling, Springer-Verlag, Berlin (1992)

Schekkerman, J.; How to Survive in the Jungle of Enterprise Architecture Frameworks. Trafford Publishing (2004)

Spadoni, M.; Distributed client/server Architecture for CIMOSA-Based Enterprise Components. Computers in industry: 55(3), 239-253 (2004)

Tang, A.; J. Han y P. Chen; A comparative analysis of Architecture Frameworks. Technical Report: SUTIT-TR2004.01 CeCSES Centre Report: SUT.CeCSES-TR001 (fecha de consulta Mayo 18, 2006; www.it.swin.edu.au/centres/TechnicalReports/2004/SUTIT-TR2004.01.pdf ) (2004)

Vernadat F. ; Enterprise Modelling and Integration. Principles and Aplication, Chapman and Hall. (1996) 